\title{
Bone morphogenetic protein 7 upregulates the expression of nestin and glial fibrillary acidic protein in rats with cerebral ischemia-reperfusion injury
}

\author{
RUI ZHANG ${ }^{1}$, HAITAO PEI ${ }^{2}$, LIJUAN RU ${ }^{2}$, HONGYUN LI $^{2}$ and GUANGYI LIU ${ }^{1}$ \\ ${ }^{1}$ Institute of Cerebrovascular Diseases; ${ }^{2}$ Department of Emergency Neurology, The Affiliated Hospital \\ of Qingdao University Medical College, Qingdao, Shandong 266003, P.R. China
}

Received July 8, 2013; Accepted August 23, 2013

DOI: $10.3892 /$ br.2013.164

\begin{abstract}
Bone morphogenetic protein 7 (BMP7) is a member of the transforming growth factor- $\beta$ (TGF- $\beta$ ) superfamily and was initially identified as a protein that may induce bone and cartilage growth in the bone matrix. The present study was conducted in order to investigate the effect of BMP7 on the expression of nestin and glial fibrillary acidic protein (GFAP) in the brain tissue of rats after cerebral ischemia-reperfusion injury. A total of 40 adult healthy male Sprague-Dawley rats were used in this study, of which 10 randomly received a sham operation and the remaining 30 were subjected to a $2-\mathrm{h}$ ischemia and 24-h reperfusion by ligation of the left external and internal carotid arteries. Twenty successfully modeled rats were equally randomized into the treatment and control groups. The rats in the treatment group were intervened with $250 \mu 1 \mathrm{BMP} 7(0.1 \mathrm{mg} / \mathrm{kg})$ via tail vein injection, whereas the rats in the control and sham operation groups were injected with an equal volume of sterile water for injection. Neurological deficits were evaluated by the Bederson's method at $24 \mathrm{~h}$ after ischemia-reperfusion and the brain infarct volume was assessed by 2,3,5-triphenyl tetrazolium chloride coloring. The neuronal apoptosis was assessed by terminal deoxynucleotidyl transferase-mediated biotinylated deoxyuridine triphosphate nick end-labelling (TUNEL) staining and the expression of nestin and GFAP in the three groups was analyzed by immunohistochemistry. Bederson's score $(\mathrm{t}=4.66, \mathrm{P}<0.01)$ and focus
\end{abstract}

Correspondence to: Dr Haitao Pei, Department of Emergency Neurology, The Affiliated Hospital of Qingdao University Medical College, 16 Jiangsu Road, Qingdao, Shandong 266003, P.R. China E-mail: zztg@163.com; peihtao@163.com

Abbreviations: GFAP, glial fibrillary acidic protein; TGF- $\beta$, transforming growth factor- $\beta$; TTC, tetrazolium chloride; BMP7, bone morphogenetic protein 7 ; $\mathrm{MCAO} / \mathrm{R}$, middle cerebral artery occlusion/reperfusion; TUNEL, terminal deoxynucleotidyl transferasemediated biotinylated deoxyuridine triphosphate nick end-labelling

Key words: bone morphogenetic protein 7, nestin, glial fibrillary acidic protein, cerebral ischemia-reperfusion injury, rats infarction $(\mathrm{t}=6.98, \mathrm{P}<0.01)$ were lower in the BMP7 treatment group compared to those in the control group. In addition, the number of TUNEL-positive cells in the treatment group was lower compared to that in the control group $(\mathrm{P}<0.01)$. Compared to the control group, the expression of nestin and GFAP was enhanced in the BMP7 treatment group $(\mathrm{P}<0.01)$. Therefore, BMP7 may upregulate the expression of nestin and GFAP and promote neural regeneration to protect animals against ischemia-reperfusion injury.

\section{Introduction}

Bone morphogenetic protein 7 (BMP7) is a member of the transforming growth factor- $\beta$ (TGF- $\beta$ ) superfamily and was initially identified as a protein that may induce bone and cartilage growth in the bone matrix. Previous studies suggested that BMP7 acts on the skeletal system and is also expressed in the neural tissues of adult animals together with its receptor (1), playing an important role in the process of neural histogenesis (2). It was previously confirmed that the injection of BMP7 into the cisterns prior to focal cerebral ischemia in adult rats significantly improved motor function and reduced the volume of the cerebral infarct. The injection of BMP7 into the cisterns at $24 \mathrm{~h}$ following ischemia did not reduce the volume of the cerebral infarct, although it significantly promoted the motor function recovery of the affected rat limbs $(3,4)$. Chou et al $(5)$ reported that BMP7 promoted the proliferation of neural progenitor cells in the infarction region-contralateral subventricular zone of middle cerebral artery occlusion (MCAO) rats and observed that the proliferated neural progenitor cells migrated to the cortex of the region surrounding the infarction through the corpus callosum. Nestin is considered to be one of the markers of neural precursor cells (6) and nestin-positive cells may be considered as neural progenitor cells. The glial fibrillary acidic protein (GFAP) is a specific cytoskeletal protein mainly present in astrocytes and it is considered as a marker of astrocyte maturation (7). The astrocytes are activated at the time of cerebral ischemia-reperfusion injury, reflected by hypertrophic and edematous astrocyte bodies, increased and extended apoptosis and enhanced GFAP expression demonstrated by immunohistochemical staining. The significance of GFAP may be closely associated with the 
repair of the central lesion. The aim of this experiment was to investigate the effect of BMP7, injected via the caudal vein at $2 \mathrm{~h}$ following focal cerebral ischemia, on the expression of nestin and GFAP after cerebral ischemia-reperfusion injury in adult rats and assess the neuroprotective effect of BMP7 following cerebral ischemia-reperfusion injury, as well as its underlying mechanism.

\section{Materials and methods}

Animal model. A total of 40 adult healthy male Sprague-Dawley rats of clean grade, weighing 230-250 g, were provided by the Qingdao Experimental Animal Center (2009SCXK0010). The animals were kept in the laboratory at room temperature $\left(23 \pm 2^{\circ} \mathrm{C}\right)$ under natural illumination during an acclimatization period of 1 week prior to the experiment. Ten rats randomly received a sham operation and the remaining 30 rats were subjected to a 2-h ischemia and 24-h reperfusion by ligation of left external and internal carotid arteries (8). The Bederson's scale (9) was adopted to evaluate the neurological deficits after the postoperative animals regained consciousness and 20 animals (10 unsuccessful animals were excluded) with a Bederson's score of $\geq 2$ were equally randomized into the BMP7 treatment and control groups.

This study was reviewed and approved by our Institutional Animal Care and Use Committee.

Drug administration. BMP7 (BS0504P; Beijing Biosynthesis Biotechnology Co. Ltd., Beijing, China), as the investigational drug, was dissolved in sterile water for injection. The rats in the treatment group were intervened with $250 \mu 1 \mathrm{BMP} 7$ $(0.1 \mathrm{mg} / \mathrm{kg})$ via tail vein injection using a microsyringe immediately after the $2 \mathrm{~h}$-ischemia reperfusion, whereas the rats in the control and sham operation groups were injected with an equal volume of sterile water for injection.

Neurological deficit scoring. The Bederson's scale (9) was used for neurological deficit scoring after the animals had regained consciousness: grade 0 , no signs of neurological deficits; grade 1 , rats with infarction flexed the forelimb contralateral to the injured hemisphere when their tails were raised, while extending the normal forelimb to the ground; grade 2, apart from the signs of grade 1 , there was a significantly decreased resistance to lateral push without circling; grade 3, rats exhibited the same behavior as in grade 2, with circling.

Triphenyl tetrazolium chloride (TTC) staining. Five animals were selected from each group and were anesthetized by intraperitoneal injection of $0.3 \mathrm{ml} 10 \%$ chloral hydrate at $24 \mathrm{~h}$ after reperfusion. The rats were decapitated and the brains were removed. Five continuous coronal brain sections were obtained at 2-mm intervals, starting $2 \mathrm{~mm}$ from the polus frontalis. The sections were immersed in 1\% TTC solution and placed in a $37^{\circ} \mathrm{C}$ incubator for $30 \mathrm{~min}$. The staining effect was as follows: normal brain tissue appeared red, whereas infarcted brain tissue appeared more pale. After capturing images of the stained brain tissue sections, the Image-Pro Plus image analysis system, version 6.0 (Fryer Co, Chicago, IL, USA) was used to calculate the infarction area and the total brain section area in each brain sample. The following formula was used to calculate the infarction area per brain section and the total brain section area and, subsequently, the percentage of cerebral infarct volume of each rat: percentage of cerebral infarct volume $=($ infarction area of brain section/total area of brain section) $\mathrm{x} 100 \%$.

Preparation of paraffin sections. Five animals were selected from each group, anesthetized with an intraperitoneal injection of $0.3 \mathrm{ml} 10 \%$ chloral hydrate and positioned in dorsal recumbency. Physiological saline and $4 \%$ formaldehyde solution were administered to perform cardiac perfusion fixation. The rats were decapitated and their brains were removed. Ethanol was used for dehydration at the conventional gradient, xylene for vitrification and paraffin for embedding. Continuous coronal $2-\mu \mathrm{m}$ sections were obtained from the posterior of the optic chiasma, mounted on microscopic slides, treated with polylysine and stored for later use.

Apoptosis detection. The sections were routinely deparaffinized and hydrated. After washing with distilled water, apoptosis assessment was conducted according to the instructions in the terminal deoxynucleotidyl transferase (TdT)-mediated biotinylated deoxyuridine triphosphate nick end-labelling (TUNEL) apoptosis test kit (Wuhan Boster Biotech. Co. Ltd., Wuhan, China). Cells with nuclei containing brown particles observed under a light microscope were considered as positive. In some sections, TdT was replaced by $0.01 \mathrm{~mol} / \mathrm{l}^{-1}$ phosphate-buffered saline (PBS), which yielded no positive reaction. Four sections per rat were obtained and positive cells were counted in four random visual fields in the cortex, corpus striatum and hippocampus under high-power magnification.

Immunohistochemical staining. The sections mentioned above were routinely deparaffinized and hydrated. After washing with distilled water, nestin and GFAP immunohistochemical staining was performed in compliance with the instructions (Wuhan Boster Biotech. Co. Ltd.). Cells with cytoplasm or nuclei containing brown particles under a light microscope were considered as positive. In some sections, the primary antibody was replaced with $0.01 \mathrm{~mol} / 1 \mathrm{PBS}$, which yielded no positive reaction. Four sections per rat were obtained and positive cells were counted in four random visual fields in the cortex, corpus striatum and hippocampus under a light microscope (magnification, $\mathrm{x} 400$ ) to calculate the number of positive cells per visual field.

Statistical method. SPSS statistical software, version 11.0 (SPSS Inc., Chicago, IL, USA) was adopted to conduct data analysis. Data are expressed as means \pm standard deviation. One-factor analysis of variance was adopted with a significance level $(\alpha)$ of 0.05 .

\section{Results}

Neurological deficit scores. No postoperative neuromotor dysfunction was identified in the sham operation group. The majority of the animals in the model group regained consciousness $2 \mathrm{~h}$ after the operation. The rats developed right limb dysfunction after waking up and presented with right forelimb 


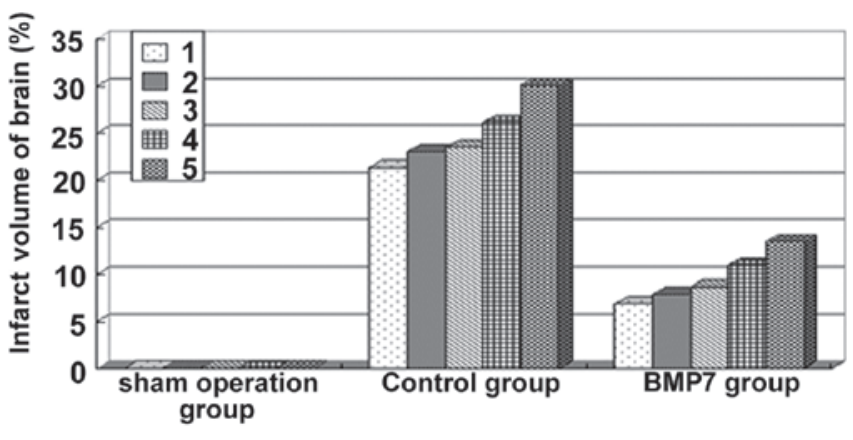

Figure 1. Percentage of brain infarct volume.

flexion when their tails were raised and suspended in mid-air. The Bederson's score in the BMP7 treatment group (1.7 \pm 0.48$)$ was significantly lower compared to that in the control group $(2.7 \pm 0.48)$, with a statistically significant difference $(\mathrm{t}=4.66$, $\mathrm{P}<0.01)$.

Cerebral infarct volume percentage in rats from each group. The TTC staining results suggested that the brains of the rats from the sham operation group were all stained red and the cerebral infarct volume percentage in the model group was significant. The cerebral infarct volume percentage in the BMP7 group $(9.57 \pm 2.67 \%)$ was significantly decreased compared to the control group $(24.81 \pm 3.41 \%)$, with a statistically significant difference $(\mathrm{t}=7.87, \mathrm{P}<0.01)$ (Fig. 1).

Number of TUNEL-positive cells. The cells were evenly distributed in the brain sections from rats in the sham operation group. Scattered TUNEL-positive cells were identified in the cerebral cortex. The number of TUNEL-positive cells in the control group was higher compared to that in the sham operation group and these cells were mainly distributed in the ischemic cortex, corpus striatum and hippocampus. The numbers of TUNEL-positive cells in the ischemic cortex, corpus striatum and hippocampus in the BMP7 treatment group were significantly lower compared to those in the control group and the differences were statistically significant $(\mathrm{t}=17.31, \mathrm{t}=12.45$ and $\mathrm{t}=17.38$, respectively; $\mathrm{P}<0.01)$. Apart from the sham operation group, the majority of TUNEL-positive cells were located in the corpus striatum, as determined by intra-group comparison, followed by the hippocampus and cortex, with a statistically significant difference $(\mathrm{t}=2.77-6.71$, $\mathrm{P}<0.05)$. The results are shown in Table I.

Nestin expression in rat brains. Nestin-positive cells were almost absent in the sham operation group, whereas increased numbers of nestin-positive cells were identified in the control group and were mainly distributed in the ischemic cortex, corpus striatum and hippocampus. The numbers of nestin-positive cells in the ischemic cortex, corpus striatum and hippocampus in the BMP7 group were significantly higher compared to those in the control group, with statistically significant differences $(t=16.03, t=8.28$ and $t=12.68$, respectively; $\mathrm{P}<0.01$ ). Except for the sham operation group, the majority of nestin-positive cells were located in the ischemic cortex, as determined by intra-group comparison, followed
Table I. TUNEL-positive cells in brain tissue (means \pm standard deviation per visual field).

\begin{tabular}{lcccc}
\hline Groups & $\mathrm{n}$ & Cortex & $\begin{array}{c}\text { Corpus } \\
\text { striatum }\end{array}$ & Hippocampus \\
\hline Sham & 5 & $1.8 \pm 0.84$ & $3.2 \pm 0.71$ & $3.0 \pm 0.84$ \\
Control & 5 & $13.4 \pm 1.14^{\mathrm{a}}$ & $17.8 \pm 1.48^{\mathrm{a}}$ & $15.4 \pm 1.14^{\mathrm{a}}$ \\
BMP7 & 5 & $3.6 \pm 0.55^{\mathrm{b}}$ & $7.4 \pm 1.14^{\mathrm{b}}$ & $5.0 \pm 0.70^{\mathrm{b}}$ \\
\hline
\end{tabular}

${ }^{\mathrm{a}} \mathrm{P}<0.01$ vs. sham group; ${ }^{\mathrm{b}} \mathrm{P}<0.01$ vs. control group. TUNEL, terminal deoxynucleotidyl transferase-mediated biotinylated deoxyuridine triphosphate nick end-labelling; BMP, bone morphogenetic protein.

Table II. Nestin-positive cells in brain tissue (means \pm standard deviation per visual field).

\begin{tabular}{lcccc}
\hline Groups & $\mathrm{n}$ & Cortex & $\begin{array}{c}\text { Corpus } \\
\text { striatum }\end{array}$ & Hippocampus \\
\hline Sham & 5 & $2.8 \pm 0.84$ & $2.8 \pm 0.84$ & $2.4 \pm 0.55$ \\
Control & 5 & $8.6 \pm 0.55^{\mathrm{a}}$ & $7.2 \pm 1.30^{\mathrm{a}}$ & $5.8 \pm 0.84^{\mathrm{a}}$ \\
BMP7 & 5 & $15.8 \pm 0.84^{\mathrm{b}}$ & $13.6 \pm 1.14^{\mathrm{b}}$ & $12.0 \pm 0.70^{\mathrm{b}}$ \\
\hline
\end{tabular}

${ }^{\mathrm{a}} \mathrm{P}<0.01$ vs. sham group; ${ }^{\mathrm{b}} \mathrm{P}<0.01$ vs. control group. $\mathrm{BMP}$, bone morphogenetic protein.

Table III. GFAP-positive cells in brain tissue (means \pm standard deviation per visual field).

\begin{tabular}{lcccc}
\hline Groups & $n$ & Cortex & $\begin{array}{c}\text { Corpus } \\
\text { striatum }\end{array}$ & Hippocampus \\
\hline Sham & 5 & $4.0 \pm 1.0$ & $2.8 \pm 0.84$ & $7.0 \pm 0.71$ \\
Control & 5 & $9.4 \pm 1.14^{\mathrm{a}}$ & $6.0 \pm 0.71^{\mathrm{a}}$ & $13.2 \pm 0.84^{\mathrm{a}}$ \\
BMP7 & 5 & $15.4 \pm 0.84^{\mathrm{b}}$ & $14.2 \pm 0.55^{\mathrm{b}}$ & $17.0 \pm 0.71^{\mathrm{b}}$ \\
\hline
\end{tabular}

${ }^{\mathrm{a}} \mathrm{P}<0.01$ vs. sham group; ${ }^{\mathrm{b}} \mathrm{P}<0.01$ vs. control group. GFAP, glial fibrillary acidic protein; BMP, bone morphogenetic protein.

by the corpus striatum and hippocampus, with statistically significant differences $(\mathrm{t}=2.22-7.77, \mathrm{P}<0.05)$. The results are shown in Fig. 2 and Table II.

GFAP expression in in rat brains. GFAP-positive cells were rare in the sham operation group (concentration of GFAP antibody, 1:150), whereas increased numbers of GFAP-positive cells were identified in the control group and were mainly distributed in the ischemic cortex, corpus striatum and hippocampus. The numbers of GFAP-positive cells in the ischemic cortex, corpus striatum and hippocampus in the BMP7 group were significantly higher compared to those in the control group, with statistically significant differences $(\mathrm{t}=9.47, \mathrm{t}=20.42$ and $t=7.73$, respectively; $P<0.01$ ). Apart from the sham operation group, the majority of GFAP-positive cells were located in 

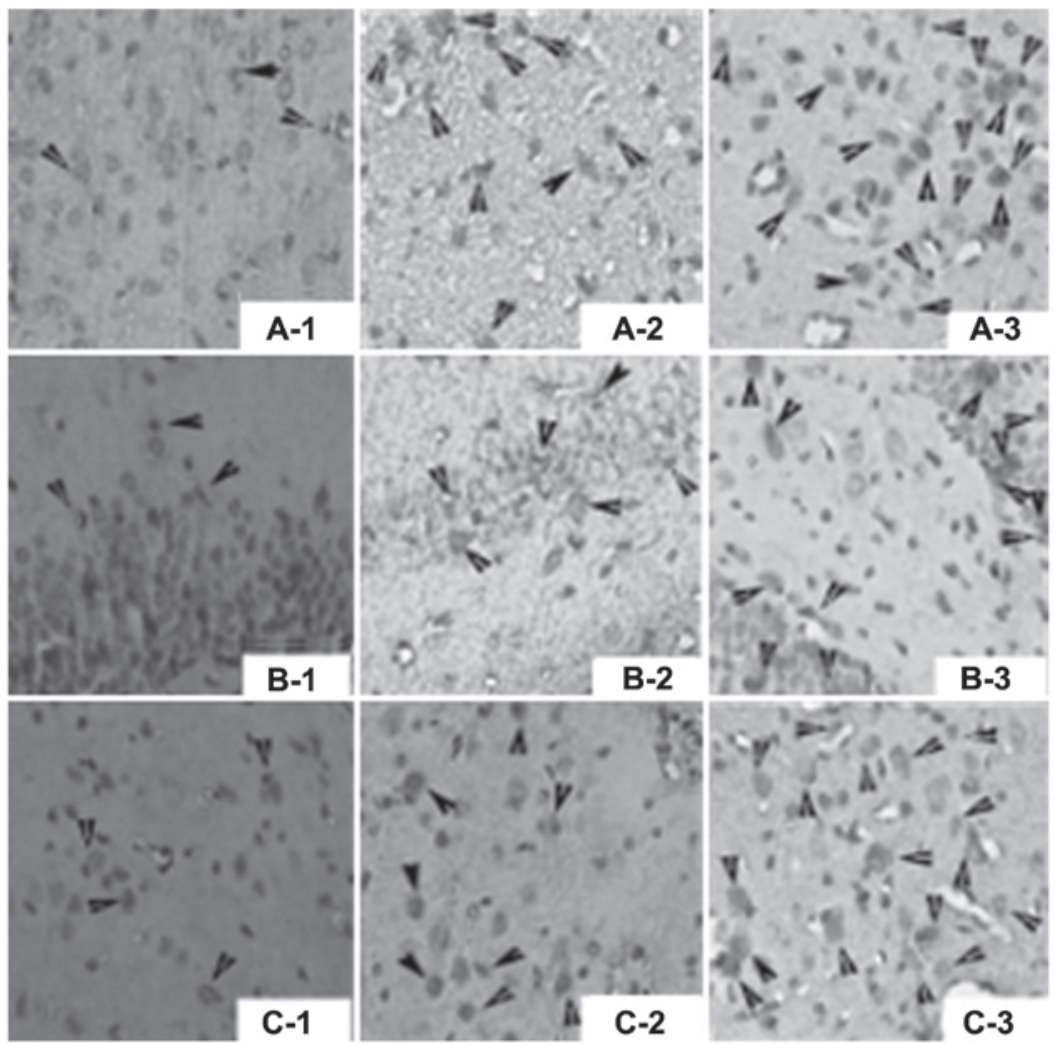

Figure 2. Nestin-positive cells (arrows) shown by immunohistochemical assay (magnification, $x 400$ ). (A) Nestin-positive cells in the cortex in: A-1, sham operation group; A-2, control group; and A-3, bone morphogenetic protein 7 (BMP7) group. (B) Nestin-positive cells in the hippocampus in: B-1, sham operation group; B-2, control group; and B-3, BMP7 group. (C) Nestin-positive cells in the striatum in: C-1, sham operation group; C-2, control group; and C-3, BMP7 group.
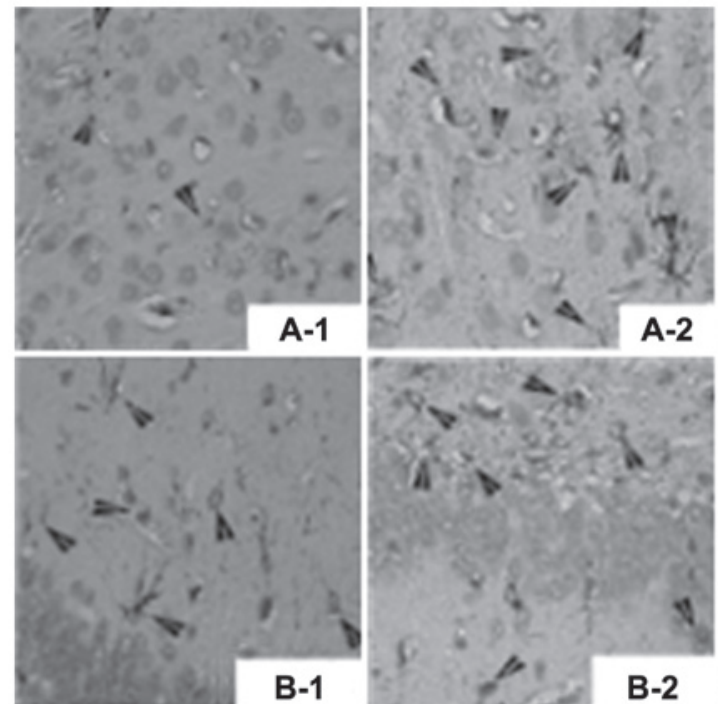

A-2
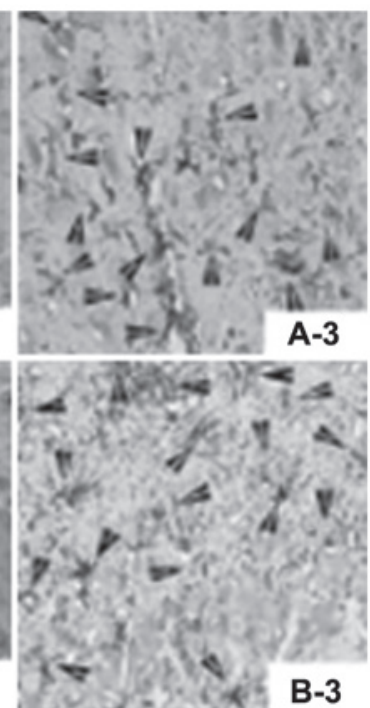

B-2
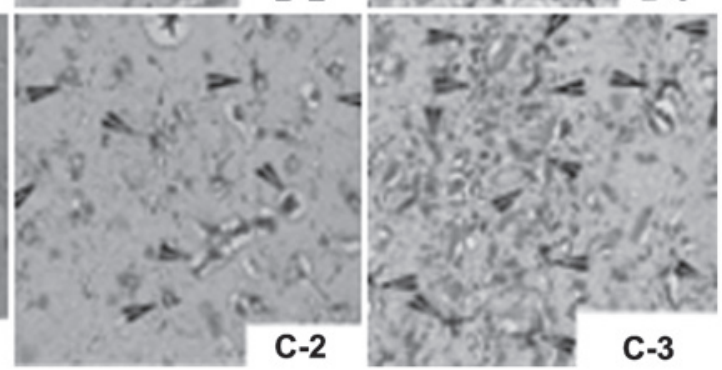

Figure 3. Glial fibrillary acidic protein (GFAP)-positive cells (arrows) shown by immunohistochemical assay (magnification, x400). (A) GFAP-positive cells in the cortex in: A-1, sham operation group; A-2, control group; and A-3, bone morphogenetic protein 7 (BMP7) group. (B) GFAP-positive cells in the hippocampus in: B-1, sham operation group; B-2, control group; and B-3, BMP7 group. (C) GFAP-positive cells in the striatum in: C-1, sham operation group; $\mathrm{C}-2$, control group; and C-3, BMP7 group. 
the hippocampus, as determined by intra-group comparison, followed by the ischemic cortex and corpus striatum, with statistically significant differences $(\mathrm{t}=2.67-14.64, \mathrm{P}<0.05)$. The results are presented in Fig. 3 and Table III.

\section{Discussion}

Neurogenesis remains active in the brain of adult animals and its main function is to replace the apoptotic neurons in the specific encephalic region. For example, the granular cells of the dentate gyrus regularly undergo apoptosis, whereas neural progenitor cells proliferate with the same rate to maintain a stable number of granulosa cells (10). It was demonstrated that when an infarction develops in the unilateral cortex and corpus striatum in rats with middle cerebral artery embolism, the neural progenitor cells in the subventricular zone and dentate gyrus increasingly proliferate, migrate to the surroundings of the infarcted region and differentiate into neurons and astrocytes (11). Therefore, the injured brain cells in patients with apoplexy are expected to exhibit neural regenerative potential. It was previously confirmed that BMP7 may promote DNA synthesis in brain cells cultured in vitro and simultaneously promote the dendrite growth of sympathetic neurons cultured in vitro (12). In rats without BMP type I receptors, the expression of netrin, a nerve growth factor that induces axon growth, is inhibited (13). Therefore, the activation of BMP signal transduction plays an important role in cell proliferation and neural regeneration.

Nestin is a type IV intermediate filament protein, which is distributed in the cytoplasm, is mainly expressed in the stem cells of the neuroepithelium and participates in the construction of the cytoskeleton. When the nerve cell migration is almost completed, the nestin expression begins to decrease and stops with cell maturation. Nestin-positive cells cultured in vitro may differentiate into the precursor cells of neurons and gliocytes; therefore, nestin is considered to be an important protein marker of neural stem cells. Nestin-positive cells are mainly distributed in blood vessels, submeningeal spaces, choroid plexus and among ependymal epithelial cells, as well as in the subependymal region (14). Following ischemic injury, the expression of nestin is significantly increased. In addition to the regions mentioned above, nestin expression is also detected in the central ischemic region and its surroundings. The results of our experiment demonstrated that, compared to the same region in the control group, BMP7 injection via the caudal vein after a 2-h MCAO ischemia-reperfusion was able to increase the expression of nestin in the injured cortex, corpus striatum and hippocampal dentate gyrus in rats $24 \mathrm{~h}$ after suffering a stroke. Therefore, BMP7 may exert a neuroprotective effect by promoting neural regeneration.

Astrocytes are widely distributed in the central nervous system. Apart from their supporting and nutritional functions, astrocytes also play an important role in nerve tissue repair and regeneration. The application of immunohistochemical staining for GFAP may reflect the morphological changes exhibited by astrocytes $(15,16)$. GFAP is rarely expressed under physiological conditions and is mainly involved in the structure of neurons and the regulation of substance metabolism, as well as cytoskeleton recombination and the functioning of the blood-brain barrier; it is also involved in intercellular signal transduction pathways (17). Nawashiro et al (18) established a cerebral ischemia model by using wild-type mice lacking GFAP and demonstrated that the cortex infarction volume was significantly increased compared to the control group, indicating that astrocytes and GFAP may exert a neuroprotective effect on brain tissue suffering ischemia-reperfusion injury. GFAP is expressed by a large number of astrocytes at the time of cerebral ischemia, which may be associated with the phagocytosis of harmful extracellular neurohumors, the maintenance of intracephalic environment stability and neuron survival and plasticity repair (19). The present experiment confirmed that GFAP expression was increased after cerebral ischemia and was mainly distributed in the ischemic cortex, corpus striatum and hippocampus. BMP7 may upregulate the expression of GFAP after cerebral ischemia and provide protection for ischemic and anoxic neurons.

In conclusion, BMP7 may upregulate the expression of nestin and GFAP and promote neural regeneration, protecting animal cells against ischemic injury.

\section{Acknowledgements}

This study was supported by grant-in-aids for the Natural Science Foundation of Shandong province (grant no. ZR2009CM121).

\section{References}

1. Chen HL, Lein PJ, Wang JY, et al: Expression of bone morphogenetic proteins in the brain during normal aging and in 6-hydroxydopamine-lesioned animals. Brain Res 994: 81-90, 2003.

2. Qin L, Wine-Lee L, Ahn KJ, et al: Genetic analyses demonstrate that bone morphogenetic protein signaling is required for embryonic cerebellar development. J Neurosci 26: 1896-1905, 2006.

3. Kawamata T, Ren J, Chan TC, et al: Intracisternal osteogenic protein-1 enhances functional recovery following focal stroke. Neuroreport 9: 1441-1445, 1998.

4. Schallert T, Fleming SM, Leasure JL, et al: CNS plasticicy and assessment of forelimb sensorimotor outcome in unilateral rat models of stroke, cortical ablation, parkinsonism and spinal cord injury. Neuropharmacology 39: 777-787, 2000.

5. Chou J, Harvey BK, Chang CF, et al: Neuroregenerative effects of BMP7 after stroke in rats. J Neurol Sci 240: 21-29, 2006.

6. Almazan G, Vela JM, Molina-Holgado E, et al: Re-evaluation of nestin as a marker of oligodendrocyte lineage cells. Microsc Res Tech 52: 753-756, 2001.

7. Gomes FC, Paulin D and Moura Neto V: Glial fibrillary acidic protein (GFAP): modulation by growth factors and its implication in astrocyte differentiation. Braz J Med Biol Res 32: 619-631, 1999.

8. Longa EZ, Weinstein PR, Carlson S, et al: Reversible middle cerebral artery occlusion without craniectomy in rats. Stroke 20: 84-91, 1989.

9. Bederson JB, Pitts LH, Tsuji M, et al: Rat middle cerebral artery occlusion: evaluation of the model and development of a neurologic examination. Stroke 17: 472-476, 1986.

10. Wiltrout C, Lang B, Yan Y, et al: Repairing brain after stroke: a review on post-ischemic neurogenesis. Neurochem Int 50: 1028-1041, 2007.

11. Zhang PB, Liu Y, Li J, et al: Ependymal/subventricular zone cells migrate to the peri-infarct region and differentiate into neurons and astrocytes after focal cerebral ischemia in adult rats. Academic Journal of the First Medical College of PLA 25: 1201-1206, 2005

12. Lein P, Johnson M, Guo X, et al: Osteogenic protein-1 induces dendritic growth in rat sympathetic neurons. Neuron 15: 597-605, 1995.

13. Liu J, Wilson $\mathrm{S}$ and Reh T: BMP receptor $\mathrm{lb}$ is required for axon guidance and cell survival in the developing retina. Dev Biol 256: 34-48, 2003. 
14. Liu PC, Lu SD, Huang YL, et al: The expression of nestin in ischemia-injured brain of adult rat. Acta Physiologica Sinica 54: 294-299, 2002 (In Chinese).

15. Chen HX, Zhang LM, Zhang YZ, et al: Effect of agmatine on the neurons and astrocytes in hippocampus of chronically stressed rats. Chin Pharmacol Bull 25: 21-25, 2009.

16. Zhang JJ: Advances in astrocytes. Acta Pharmacologica Sinica 22: 788-791, 2006 (In Chinese).

17. Timmer M, Cesnulevicius K, Winkler C, et al: Fibroblast growth factor (FGF)-2 and FGF receptor 3 are required for the development of the substantia nigra, and FGF-2 plays a crucial role for the rescue of dopaminergic neurons after 6-hydroxydopamine lesion. J Neurosci 27: 459-471, 2007.
18. Nawashiro H, Brenner M, Fukui S, et al: High susceptibility to ischemia in GFAP-null mice. J Cereb Blood Flow Metab 20: 1040-1044, 2000.

19. Liu D, Smith CL, Barone FC, et al: Astrocytic demise precedes delayed neuronal death in focal ischemic rat brain. Brain Res Mol Brain Res 68: 29-41, 1999. 